\title{
Neonatal Pneumothorax in Late Preterm and Full-Term Newborns with respiratory Distress: A Single-Center Experience
}

Eun-Ah Kim, MD ${ }^{1,2}$, Jae-Hun Jung, MD ${ }^{1,2}$, Sang-Yoon Lee, $\mathrm{MD}^{1,2}$, Sook-Hyun Park, $\mathrm{MD}, \mathrm{PhD}^{1,2}$, and Ji Sook Kim, $\mathrm{MD}$, $\mathrm{PhD}^{1,2}$

${ }^{1}$ Department of Pediatrics, School of Medicine, Kyungpook National University, Daegu, Korea

${ }^{2}$ Division of Neonatology, Department of Pediatrics, Kyungpook National University Chilgok Hospital, Daegu, Korea

\section{ABSTRACT}

Purpose: To evaluate the incidence rate, clinical characteristics, and perinatal outcomes of pneumothorax in late preterm and full-term newborns with respiratory distress and analyze the risk factors associated with pneumothorax.

Methods: Infants born at $\geq 34$ weeks' gestation with respiratory distress and pneumothorax admitted between February 2014 and December 2020 were enrolled in this study. The pneumothorax group $(n=36)$ was matched to the control group $(n=144)$ in a 1:4 ratio, based on gestational age and birth weight. Risk factors were identified using logistic regression analysis with backward stepwise selection.

Results: The incidence of pneumothorax during the study period was $1.36 \%(38 / 2,788)$. All patients were diagnosed with pneumothorax within 48 hours after birth, and increased oxygen demand was the most common symptom. The proportion of mortality and perinatal morbidity, such as intraventricular hemorrhage $\geq$ grade 3 , was significantly higher in the pneumothorax group than in the control group. The risk factors associated with pneumothorax were the need for positive pressure ventilation in the delivery room (odds ratio [OR], 3.40; 95\% confidence interval [CI], 1.26 to 9.12; $P=0.015$ ) and a higher fraction of inspired oxygen to achieve an oxygen saturation of $\geq 90 \%$ on admission (OR, $1.06 ; 95 \%$ CI, 1.03 to $1.09 ; P<0.001)$.

Conclusion: Pneumothorax should be suspected in late preterm and full-term newborns with respiratory distress within the first 3 days of life. Based on these risk factors, early diagnosis can reduce perinatal mortality and morbidity.

Key Words: Infant, newborn; Pneumothorax; Epidemiology; Risk factors

\section{INTRODUCTION}

Pneumothorax, a common air leak syndrome in the neonatal period, is defined as
Received: 1 January 2022

Revised: 14 February 2022

Accepted: 20 February 2022

Correspondence to: Ji Sook Kim, MD, $\mathrm{PhD}$

Division of Neonatology, Department of Pediatrics, Kyungpook National University Children's Hospital, School of Medicine, Kyungpook National University, 807 Hoguk-ro, Buk-gu, Daegu 41404, Korea Tel: +82-53-200-2750

Fax: $+82-53-200-2029$

E-mail: jisook.kim.neo@gmail.com

Copyright(c) 2022 By Korean Society of Neonatology

This is an Open-Access article distributed under the terms of the Creative Commons At tribution Non-Commercial License (http:// creativecommons.org/licenses/by-nc/4.0), which permits unrestricted non-commercial use, distribution, and reproduction in any medium, provided the original work is properly cited. 
abnormal air accumulation between the visceral and parietal pleura. It can increase intrathoracic pressure, resulting in partial or complete collapse of the lung on the affected side. The clinical manifestation of pneumothorax depends on the degree of collapse of the lung, which can impair pulmonary gas exchange and hemodynamic stability and may result in a life-threatening condition. Pneumothorax occurs more frequently in newborns than in any other age group, with an incidence rate of $1 \%$ to $2 \%$ in the general population and $6 \%$ to $7 \%$ in very low birth weight (VLBW) infants with a birth weight of less than $1,500 \mathrm{~g}^{1-}$ 3). Moreover, symptomatic pneumothorax occurs in $0.08 \%$ of all live births and $5 \%$ to $7 \%$ of VLBW infants ${ }^{4}$.

Despite advances in neonatal intensive care, such as the use of antenatal steroids, surfactant replacement therapy, and lungprotective ventilator strategies, pneumothorax remains a com mon respiratory complication that contributes to an increase in perinatal morbidities, including intraventricular hemorrhage (IVH), bronchopulmonary dysplasia (BPD), and mortality, es pecially in preterm infants and critically ill newborns ${ }^{5-7)}$. Although prematurity and respiratory distress syndrome (RDS) have been considered important contributing factors associated with pneumothorax, pneumothorax frequently occurs in all newborns who have underlying lung disease or require either noninvasive or invasive mechanical ventilation ${ }^{8,9}$. Late preterm and term newborns with neonatal pneumothorax are usually asymptomatic, but patients with respiratory distress tend to be symptomatic, resulting in acute respiratory failure or life-threatening condition ${ }^{1)}$. Pneumothorax occurring in term infants with respiratory distress is also associated with increased morbidities and length of hospitalization ${ }^{10)}$. Many previous studies on neonatal pneumothorax have focused on early preterm new. borns; therefore, further studies on the epidemiology, clinical characteristics, and risk factors of pneumothorax in late preterm and full-term newborns are required.

This study aimed to evaluate the incidence of pneumothorax in late preterm and full-term newborns with respiratory distress and compare the clinical characteristics, perinatal morbidities, and mortality between late preterm and full-term newborns with and without pneumothorax during hospitalization. Moreover, we aimed to identify the risk factors associated with pneumothorax in late preterm and full-term newborns with respiratory distress.

\section{MATERIALS AND METHODS}

\section{Study population and design}

We retrospectively reviewed the electronic medical charts of all newborns admitted to the neonatal intensive care unit(NICU) of Kyungpook National University Chilgok Hospital (KNUCH) between February 2014 and December 2020. This retrospective case-control study was approved by the Institutional Review Board of KNUCH, with a waiver of consent (2021-10-003).

Neonatal pneumothorax was defined as abnormal air accumulation between the visceral and parietal pleura within 28 days after birth or at 44 weeks of corrected gestational age (GA). Pneumothorax was classified as spontaneous/secondary, unilateral/bilateral, and tension/non-tension pneumothorax. Spontaneous pneumothorax was defined as pneumothorax that developed without apparent causes, including predisposing factors (intubation or positive pressure ventilation [PPV]) and underlying lung diseases, while secondary pneumothorax with traumatic injury to the pleura and underlying lung diseases ${ }^{11)}$. Underlying lung diseases included RDS, transient tachypnea of the newborn (TTN), meconium aspiration syndrome (MAS), congenital pneumonia, and congenital lung hypoplasia. Tension pneumothorax was defined as compromised cardiopulmonary function with a mediastinal shift in unilateral or diaphragmatic depression in bilateral pneumothorax ${ }^{11)}$. The treatment of neo natal pneumothorax in the NICU of KNUCH is as follows: (1) close observation with or without $100 \%$ supplemental oxygen administration, (2) needle aspiration, and (3) intercostal drain age (closed thoracostomy). Management strategies for neonatal pneumothorax depend on the hemodynamic stability of the infants. Newborns with asymptomatic pneumothorax or mild respiratory distress were closely observed with or without administration of $100 \%$ supplemental oxygen. If newborns had tension pneumothorax or aggravated cardiopulmonary symptoms, needle aspiration or intercostal drainage was performed immediately by experienced thoracic surgeons or neonatologists. The intercostal drainage was removed if the patient showed improvement in symptoms and signs of pneumothorax clinically and radiologically.

The inclusion criteria for the case group (pneumothorax group) were as follows: (1) infants with a GA of $\geq 34$ weeks and admitted to the NICU due to respiratory distress and (2) infants diagnosed with pneumothorax on chest radiograph during hospitalization. Infants with life-threatening congenital malfor- 
mations were excluded from this study. Four controls matched for GA and birth weight were selected from infants with a GA of $\geq 34$ weeks who were admitted to the NICU of KNUCH due to respiratory distress during the study period. We analyzed the incidence rate and described the clinical manifestations at the onset and management of the pneumothorax group. We compared demographic and clinical characteristics, resuscitation in the delivery room, respiratory support on admission, perinatal morbidities, and mortality between the pneumothorax and control groups. We also evaluated the risk factors associated with pneumothorax in newborns born with a GA $\geq 34$ weeks and respiratory distress.

\section{Variables}

The demographic and clinical characteristics were as follows: GA based on the maternal last menstrual period, modified Ballard score, obstetric ultrasonography results, birth weight, Apgar score at 1 and 5 minutes, delivery mode, gender, small for gestational age (SGA), maternal premature rupture of membranes, oligohydramnios, pregnancy-induced hypertension, gestational diabetes mellitus, and the use of antenatal steroids ${ }^{12)}$. SGA is defined as a birth weight below the 10th percentile for $\mathrm{GA}^{13)}$. Oligohydramnios refers to a decrease in amniotic fluid volume for GA, along with an amniotic fluid index $<5 \mathrm{~cm}^{14)}$.

Data on resuscitation in the delivery room and respiratory support on admission were collected. Neonatal resuscitation in the delivery room included PPV, intubation, chest compression, and continuous positive airway pressure(CPAP) during transport to the NICU. Respiratory support on admission included a highflow nasal cannula (HFNC), nasal CPAP, mechanical ventilation, fraction of inspired oxygen $\left(\mathrm{FiO}_{2}\right)$ to achieve an oxygen saturation $\left(\mathrm{SPO}_{2}\right)$ of $\geq 90 \%$, and surfactant administration.

The clinical features and respiratory support in infants with pneumothorax at diagnosis were as follows: onset time (hours after birth), symptoms, location (right and left), tension pneumothorax, respiratory support at onset (HFNC, nasal CPAP, mechanical ventilation, maximum positive expiratory end pressure [PEEP], maximum mean airway pressure [MAP], maximum $\mathrm{FiO}_{2}$ to achieve an oxygen saturation of $\geq 90 \%$ ), management (close observation, $100 \%$ supplemental oxygen administration, needle aspiration, intercostal drainage), and underlying pulmonary diseases such as RDS, TTN, MAS, and congenital pneumonia.

The perinatal outcomes included IVH with a Papile classifi- cation of $\geq$ grade $3^{15)}$, periventricular leukomalacia (PVL), mo derate to severe BPD defined as the requirement of supple mental oxygen or positive pressure to maintain an oxygen saturation of $\geq 92 \%$ before discharge from the NICU or at 36 weeks of corrected $\mathrm{GA}^{16)}$, necrotizing enterocolitis $\geq$ Bell's stage IIb ${ }^{17)}$, sepsis-confirmed bacterial or fungal pathogens on blood culture, retinopathy of prematurity requiring treatment, duration of hospitalization, and death before discharge.

\section{Statistical analysis}

Continuous variables are presented as means and standard deviations, whereas categorical variables are presented as numbers and percentages (\%). To compare the differences between the demographic clinical characteristics, resuscitation in the delivery room, respiratory support on admission, and perinatal outcomes between the two groups, Student's t-test and MannWhitney test were used for continuous variables, and the chisquare test and Fisher's exact test were used for categorical variables. Odds ratios (ORs) and 95\% confidence intervals (CIs) were calculated using logistic regression analysis to evaluate risk factors associated with neonatal pneumothorax. Backward stepwise selection was conducted to adjust for confounders owing to the retrospective nature of the present study. All statistical analyses were performed using SPSS version 26 (IBM Co., Armonk, NY, USA), and a $P<0.05$ was considered statistically significant.

\section{RESULTS}

\section{Study population and the incidence of neonatal pneumo thorax}

Between February 2014 and December 2020, 3,406 newborns were admitted to the NICU of KNUCH because of respiratory distress, and 2,788 had a GA of $\geq 34$ weeks. Among infants with GA $\geq 34$ weeks, 38 (1.4\%) were diagnosed with pneumothorax, and $32(1.1 \%)$ were diagnosed with symptomatic pneumothorax requiring intervention, such as high-concentration oxygen $\left(\mathrm{FiO}_{2} 100 \%\right)$, needle aspiration, and intercostal drainage. Two infants who died within 24 hours of birth due to life-threaten. ing congenital malformations were excluded. Finally, the present study included 36 infants (13 men and 23 women) with pneumothorax and 144 infants as the control group, which matched the GA and birth weight with pneumothorax cases. 
Table 1. Demographic and Clinical Characteristics of the Study Groups

\begin{tabular}{lccc}
\hline Characteristic & $\begin{array}{c}\text { Study } \\
(\mathrm{n}=36)\end{array}$ & $\begin{array}{c}\text { Control } \\
(\mathrm{n}=144)\end{array}$ & $P$-value \\
\hline Gestational age (wk) & $\begin{array}{c}37.9 \pm 2.0 \\
\left(34^{+2}-41^{+2}\right)\end{array}$ & $\begin{array}{c}37.7 \pm 1.9 \\
\left(34^{+2}-40^{+6}\right)\end{array}$ & 0.875 \\
Birth weight (g) & $3,050 \pm 587$ & $3,024 \pm 477$ & 0.940 \\
& $(1,740-4,020)$ & $(2,080-4,100)$ & \\
Apgar score at 1 minute & $7 \pm 3(0-9)$ & $7 \pm 1(4-10)$ & 0.376 \\
Apgar score at 1 minute $<7$ & $4(15.4)$ & $22(16.4)$ & 0.788 \\
Apgar score at 5 minutes & $8 \pm 3(0-10)$ & $9 \pm 1(6-10)$ & 0.947 \\
Apgar score at 5 minutes $<7$ & $4(11.1)$ & $1(0.7)$ & 0.006 \\
Cesarean section & $25(69.4)$ & $98(68.5)$ & 0.916 \\
Male sex & $11(30.6)$ & $58(40.3)$ & 0.340 \\
SGA & $4(11.1)$ & $12(8.4)$ & 0.145 \\
ROM $\geq 18$ hours & $1(2.8)$ & $3(2.1)$ & 0.594 \\
Oligohydramnios & $2(5.6)$ & $3(2.1)$ & 0.262 \\
PIH & $2(5.6)$ & $10(6.9)$ & 0.556 \\
GDM & $3(9.4)$ & $33(22.3)$ & 0.142
\end{tabular}

Values are expressed as mean \pm standard deviation (minimum-maximum) or number (\%).

Abbreviations: SGA, small for gestational age; ROM, rupture of membranes; PIH, pregnancy induced hypertension; GDM, gestational diabetes mellitus.

\section{The demographic and clinical characteristics of the pneu- mothorax and control groups}

Table 1 shows a comparison of the demographic and clinical characteristics of the pneumothorax and control groups. The mean GA and birth weight of the pneumothorax group were $37.9 \pm 2.0$ weeks and $3,060 \pm 587 \mathrm{~g}$, which were not statistically different compared with the control group $(37.7 \pm 1.9$ weeks of GA and 3,024 $\pm 477 \mathrm{~g}$ of birth weight). The proportion of Apgar scores at 5 minutes $<7$ in the pneumothorax group $(4 / 36,11.1 \%)$ was significantly higher than that in the control group (1/144, 0.7\%). Other demographic and clinical characteristics were not significantly different between the pneumothorax and control groups.

\section{The resuscitation in the delivery room and respiratory support on admission of the pneumothorax and control group}

Table 2 shows the data for resuscitation in the delivery room and respiratory support on admission in the pneumothorax and control groups. Cardiopulmonary resuscitation in the delivery room was more frequent in the pneumothorax group than in the

Table 2. Resuscitation in the Delivery Room and Respiratory Support on Admission in The Study Groups

\begin{tabular}{|c|c|c|c|}
\hline Variable & Study $(\mathrm{n}=36)$ & Control $(n=144)$ & $P$-value \\
\hline \multicolumn{4}{|l|}{ Delivery room } \\
\hline PPV & $15(41.7)$ & $23(16.1)$ & 0.001 \\
\hline Intubation & $4(11.1)$ & $3(2.1)$ & 0.031 \\
\hline Chest compression & $3(8.3)$ & 0 & 0.008 \\
\hline CPAP during the transport to NICU & $1(2.8)$ & $3(2.1)$ & 0.596 \\
\hline \multicolumn{4}{|l|}{ On admission } \\
\hline Respiratory support & & & $<0.001$ \\
\hline HFNC & $4(11.1)$ & $57(39.6)$ & \\
\hline Nasal CPAP & $14(38.9)$ & $62(43.1)$ & \\
\hline Mechanical ventilation & $18(50.0)$ & $18(12.5)$ & \\
\hline $\mathrm{FiO}_{2}$ to achieve $\mathrm{SPO}_{2} \geq 90 \%$ (\%) & $49.5 \pm 30.0(21-100)$ & $27.0 \pm 12.6(21-100)$ & $<0.001$ \\
\hline $\mathrm{FiO}_{2}>40$ to achieve $\mathrm{SPO}_{2} \geq 90 \%(\%)$ & $19(52.8)$ & $20(14.4)$ & $<0.001$ \\
\hline Surfactant use & $14(38.9)$ & $13(9.0)$ & $<0.001$ \\
\hline \multicolumn{4}{|l|}{ During hospitalization } \\
\hline Maximum PEEP $\left(\mathrm{cmH}_{2} \mathrm{O}\right)$ & $6.0 \pm 0.5(5.0-7.0)$ & $5.7 \pm 0.5(4.5-6.0)$ & 0.455 \\
\hline Maximum MAP $\left(\mathrm{cmH}_{2} \mathrm{O}\right)$ & $15.5 \pm 4.1(9.5-25.0)$ & $15.4 \pm 2.3(12-20)$ & 0.458 \\
\hline Maximum PIP $\left(\mathrm{cmH}_{2} \mathrm{O}\right)$ & $19.8 \pm 3.1(14-25)$ & $17.5 \pm 2.3(15-23)$ & 0.034 \\
\hline Maximum $\mathrm{FiO}_{2}(\%)$ & $85.4 \pm 26.6(35-100)$ & $68.7 \pm 26.5(30-100)$ & $<0.001$ \\
\hline Duration of respiratory support (d) & $10 \pm 10(1-54)$ & $7 \pm 5(0-30)$ & 0.048 \\
\hline
\end{tabular}

Values are expressed as number (\%) or mean \pm standard deviation (minimum-maximum).

Abbreviations: PPV, positive pressure ventilation; CPAP, continuous positive airway pressure; NICU, neonatal intensive care unit; HFNC, high flow nasal cannula; $\mathrm{FiO}_{2}$, fraction of inspired oxygen; $\mathrm{SPO}_{2}$, saturation of percutaneous oxygen; $\mathrm{PEEP}$, positive expiratory end pressure; MAP, mean airway pressure; PIP, peak inspiratory pressure, $\mathrm{FiO}_{2}$, fraction of inspired oxygen. 
control group; 15 (41.7\%) and 23 (16.1\%) infants received PPV in the pneumothorax and control groups, respectively $(P=0.001)$; four $(11.1 \%)$ and three $(2.1 \%)$ infants received endotracheal intubation in the pneumothorax and control groups, respectively $(P=0.041)$; and three $(8.3 \%)$ and $0(0.0 \%)$ infants underwent chest compression in the pneumothorax and control groups, respectively $(P=0.035)$.

A statistically significant difference in respiratory support on admission was observed between the pneumothorax and control groups. The majority of infants with pneumothorax (32/36, 88.9\%) required respiratory support on admission with nasal CPAP (14/36, 38.9\%) and mechanical ventilation (18/36, $50.0 \%)$, although only approximately half of the control group (80/144, 55.6\%) required respiratory support with nasal CPAP $(62 / 144,43.1 \%)$ and mechanical ventilation $(18 / 144,12.5 \%)$.

Table 3. The Clinical Features and Respiratory Support in Infants with Pneumothorax at Diagnosis

\begin{tabular}{|c|c|}
\hline Variable & Study $(n=36)$ \\
\hline Onset time (hours after birth) & $20.2 \pm 14.8(1.5-48.0)$ \\
\hline \multicolumn{2}{|l|}{ Symptoms at diagnosis } \\
\hline Tachypnea & $18(50.0)$ \\
\hline Subcostal, intercostal retraction & $6(16.7)$ \\
\hline Increased oxygen need & $31(86.1)$ \\
\hline Hypotension & $16(44.4)$ \\
\hline \multicolumn{2}{|l|}{ Location } \\
\hline Right & $23(63.9)$ \\
\hline Left & $9(25.0)$ \\
\hline Both & $4(11.1)$ \\
\hline Tension pneumothorax & $11(30.6)$ \\
\hline \multicolumn{2}{|l|}{ Respiratory support at onset } \\
\hline HFNC & $5(13.9)$ \\
\hline Nasal CPAP & $10(27.8)$ \\
\hline Mechanical ventilation & $19(52.8)$ \\
\hline Maximum PEEP, $\mathrm{cmH}_{2} \mathrm{O}$ & $6.5 \pm 1.2$ \\
\hline Maximum MAP, $\mathrm{cmH}_{2} \mathrm{O}$ & $11.8 \pm 4.2$ \\
\hline Maximum $\mathrm{FiO}_{2}, \%$ & $56.4 \pm 31.6$ \\
\hline \multicolumn{2}{|l|}{ Treatment } \\
\hline Close observation & $4(11.1)$ \\
\hline High concentrated oxygen $\left(\mathrm{FiO}_{2} 100 \%\right)$ & $8(22.2)$ \\
\hline Needle aspiration & $1(2.8)$ \\
\hline Intercostal drainage (thoracostomy) & $23(63.9)$ \\
\hline
\end{tabular}

Values are expressed as mean \pm standard deviation (minimum-maxi mum) or number (\%).

Abbreviations: HFNC, high flow nasal cannula; CPAP, continuous positive airway pressure; PEEP, positive expiratory end pressure; MAP, mean airway pressure; $\mathrm{FiO}_{2}$, fraction of inspired oxygen.
The $\mathrm{FiO}_{2}$ to achieve $\mathrm{SPO}_{2}$ of $\geq 90 \%$ of the pneumothorax group $(49.5 \% \pm 30.0 \%)$ was significantly higher than that of the control group $(27.0 \% \pm 12.6 \%)$. The use of surfactants for RDS or MAS was more frequent in infants with pneumothorax than in those without pneumothorax (14/36 [38.9\%] and 13/144 [2.0\%] in the pneumothorax and control groups, respectively).

\section{The clinical characteristics at onset of pneumothorax}

Table 3 shows the clinical features and respiratory support data for the diagnosis of pneumothorax in newborns in the pneumothorax group. Thirty-six infants were diagnosed with pneumothorax presenting with respiratory distress symptoms (tachypnea, chest retraction, nasal flaring, cyanosis), hypercar bia, or increased supplemental oxygen concentration at a mean age of $20.2 \pm 14.8$ hours of life. Among 34 (94.4\%) infants who needed respiratory support at the onset of pneumothorax, 29 (80.6\%) received CPAP (10/36, 27.8\%) and mechanical ventilation. The mean maximum PEEP and MAP at the diagnosis of pneumothorax were $6.5 \pm 1.2$ and $11.8 \pm 4.3 \mathrm{cmH}_{2} \mathrm{O}$, respectively. The mean $\mathrm{FiO}_{2}$ to achieve an oxygen saturation of $\geq 90 \%$ at the diagnosis of pneumothorax was $55.8 \% \pm 31.6 \%$. Thirty-two (88.9\%) infants had unilateral pneumothorax (right, $n=23$; left, $n=9$ ), and four (11.1\%) had bilateral pneumothorax. Eleven (30.6\%) patients experienced hemodynamic instability such as hypotension, low blood oxygen levels, and shock; consequently, they were diagnosed with tension pneumothorax. Twenty-four patients $(66.7 \%)$ required needle aspiration $(1 / 36,2.8 \%)$ and intercostal drainage (23/36, 63.9\%).

\section{The final diagnosis for respiratory distress and perinatal outcomes of the pneumothorax and control groups}

Table 4 shows a comparison of the final diagnosis and perinatal outcomes between the pneumothorax and control groups. A significant difference in underlying pulmonary diseases was observed between the pneumothorax and control groups. Infants who developed pneumothorax were predominantly diagnosed with spontaneous pneumothorax (30.6\%), RDS (27.8\%), and congenital pneumonia (16.7\%); in contrast, three-quarters of the control group were diagnosed with TTN (76.4\%).

In the comparison of perinatal outcomes, the incidence rate of grade $\geq 3 \mathrm{IVH}(P=0.039)$ was significantly higher in the pneu mothorax group than in the control group. The incidence rate of secondary pulmonary hypertension was higher in the pneu mothorax group than in the control group, but the difference 
Table 4. The Comparison of Perinatal Outcomes in Neonatal Intensive Care Unit between the Pneumothorax and Control Groups

\begin{tabular}{lccc}
\hline Variable & $\begin{array}{c}\text { Study } \\
(\mathrm{n}=36)\end{array}$ & $\begin{array}{c}\text { Control } \\
(\mathrm{n}=144)\end{array}$ & $P$-value \\
\hline Final diagnosis & $10(27.8)$ & $12(8.4)$ & 0.004 \\
RDS & $4(11.1)$ & $110(76.4)$ & $<0.001$ \\
TTN & $5(13.9)$ & $10(7.0)$ & 0.187 \\
MAS & $6(16.7)$ & $8(5.6)$ & 0.038 \\
Congenital pneumonia & $6(16.7)$ & $4(9.0)$ & 0.387 \\
Perinatal depression or asphyxia & $11(30.6)$ & 0 & - \\
Spontaneous pneumothorax & $2(5.7)$ & $1(0.7)$ & 0.099 \\
Cardiac anomaly & & & \\
Perinatal outcomes & $2(5.6)$ & 0 & 0.039 \\
IVH $\geq$ grade 3 & $2(5.6)$ & $3(2.1)$ & 0.585 \\
PVL (image confirmed) & 0 & 0 & - \\
Moderate to severe BPD & $4(11.1)$ & $5(3.5)$ & 0.080 \\
Pulmonary hypertension & 0 & 0 & - \\
NEC $\geq$ stage 2a & $1(2.8)$ & $4(2.8)$ & 0.585 \\
Culture-proven sepsis & 0 & 0 & - \\
ROP requiring treatment & $17 \pm 13(1-67)$ & $14 \pm 8(1-62)$ & 0.280 \\
Duration of hospitalization (d) & $6(16.7)$ & 0 & $<0.001$ \\
Death & & & \\
\hline
\end{tabular}

Values are expressed as number (\%) or mean \pm standard deviation (minimum, maximum).

Abbreviations: RDS, respiratory distress syndrome of newborn; TTN, transient tachypnea of the newborn; MAS, meconium aspiration syndrome; IVH, intraventricular hemorrhage; PVL, periventricular leukomalacia; BPD, bronchopulmonary dysplasia; NEC, necrotizing enterocolitis; ROP; retinopathy of prematurity.

was not statistically significant $(P=0.080)$. Other morbidities, including PVL, moderate to severe BPD, and culture-proven sepsis, were not significantly different between the pneumothorax and control groups. The duration of hospitalization was also not significantly different between the pneumothorax and control groups. Infants with pneumothorax had a significantly higher mortality rate than those in the control group $(P<0.001)$; six infants in the pneumothorax group died during the study period.

\section{The risk factors associated with pneumothorax in late preterm and full-term infants with respiratory distress}

Table 5 shows the results of logistic regression with backward selection, which was conducted to determine the risk factors associated with neonatal pneumothorax. The need for PPV during resuscitation in the delivery room was significantly associated with neonatal pneumothorax (OR, 3.40, 95\% CI, 1.26 to $9.12 ; P=0.015)$. However, the need for intubation was
Table 5. The Risk Factors Associated with Neonatal Pneumothorax in Late Preterm and Full-Term Infants with Respiratory Distress

\begin{tabular}{lccr}
\hline Variable & OR & $95 \% \mathrm{CI}$ & $P$-value \\
\hline Male sex & 0.40 & $0.14-1.14$ & 0.087 \\
Maternal PIH & 0.16 & $0.01-1.68$ & 0.126 \\
At the delivery room & & & \\
PPV & 3.40 & $1.26-9.12$ & 0.015 \\
Intubation & 2.58 & $0.27-24.39$ & 0.407 \\
On admission & & & \\
$\mathrm{FiO}_{2}$ to achieve $\geq \mathrm{SpO}_{2} 90 \%$ & 1.06 & $1.03-1.09$ & $<0.001$ \\
\hline
\end{tabular}

Abbreviations: OR, odds ratio; CI, confidence interval; $\mathrm{PIH}$, pregnancy induced hypertension; $\mathrm{PPV}$, positive pressure ventilation; $\mathrm{FiO}_{2}$, fraction of inspired oxygen; $\mathrm{SpO}_{2}$, saturation of percutaneous oxygen.

not significantly associated with neonatal pneumothorax (OR, 2.58; $95 \% \mathrm{CI}, 0.27$ to 24.39; $P=0.407$ ). A high requirement of $\mathrm{FiO}_{2}$ to achieve an $\mathrm{SPO}_{2}$ of $\geq 90 \%$ on admission was significantly associated with neonatal pneumothorax (OR, 1.06; 95\% CI, 1.03 to $1.09 ; P<0.001)$.

\section{DISCUSSION}

This study aimed to compare the epidemiologic and clinical characteristics and perinatal outcomes between newborns with and without pneumothorax among late preterm and full-term newborns with respiratory distress on admission and determine the risk factors associated with neonatal pneumothorax. No significant differences in epidemiologic characteristics were observed except for the proportion of Apgar score at 5 minutes $<7$, but statistically significant differences in relation to the need for advanced neonatal resuscitation at birth and respiratory support on admission were observed between the pneumothorax and control groups. As expected, significantly higher rates of IVH (grade $\geq 3$ ) and death during hospitalization were observed in infants with pneumothorax than in those in the control group. Based on the findings of this study, the use of PPV in the delivery room and a higher requirement of $\mathrm{FiO}_{2}$ to achieve an $\mathrm{SPO}_{2} \geq 90 \%$ on admission were associated with neonatal pneumothorax in late preterm and full-term infants with respiratory distress.

The incidence rate of neonatal pneumothorax is $0.5 \%$ to $1 \%$ in the total population of live births ${ }^{18)}$. A higher incidence rate of pneumothorax has been observed in critically ill newborns admitted to the NICU, and the incidence rate has been reported to vary from $1.5 \%$ to $13.5 \%^{18-20)}$. In the present study, the overall 
incidence rate of neonatal pneumothorax was $1.36 \%$, which was relatively low compared with that reported in previous studies. The incidence of neonatal pneumothorax is known to be associated with GA and birth weight, the proportion of elective cesarean section, and the severity of concurrent pulmonary disease $^{2,21-23)}$. Based on these findings, the differences in the incidence rates can be attributed to disparities in epidemiologic characteristics, severity of underlying lung disease in the study population, and institutional variation in ventilator strategies.

In the present study, late preterm and full-term newborns who developed pneumothorax needed advanced neonatal resuscitation, including PPV, intubation, and chest compression in the delivery room, as well as respiratory support on admission, such as nasal CPAP, mechanical ventilation, relatively high $\mathrm{FiO}_{2}$ to achieve an $\mathrm{SPO}_{2}$ of $\geq 90 \%$, and the use of surfactants, more than those without pneumothorax. These results were consistent with the findings of previous studies. A retrospective populationbased descriptive study conducted in Sweden documented that $80 \%$ of term neonates with pneumothorax required PPV and/ or endotracheal intubation at birth, as well as noninvasive and invasive respiratory support on admission ${ }^{24)}$. A cohort study in Denmark also reported that nearly all newborns received nasal CPAP or an invasive mechanical ventilator prior to the onset of pneumothorax ${ }^{22}$. Indeed, the final diagnosis of respiratory distress was significantly different between the pneumothorax and control groups in this study. Newborns with pneumothorax are commonly diagnosed with RDS and congenital pneumonia, and more than three-quarters of infants without pneumothorax are confirmed to have TTN. Previous studies have also described that RDS, MAS, and congenital pneumonia were more common in infants with pneumothorax than in those without pneumo. thorax ${ }^{24,25)}$. Some newborns with pneumothorax were diagnosed with TTN, but most cases of pneumothorax that occurred were asymptomatic and spontaneously resolved without treatment such as needle aspiration and intercostal drainage ${ }^{22)}$. These findings suggest that late preterm and full-term infants who de veloped pneumothorax after birth were more likely to experience failure to cardiopulmonary transition from intra-uterine to extrauterine environment and have serious underlying pulmonary disorders, including RDS, congenital pneumonia, or systemic conditions, including perinatal asphyxia, than those without pneumothorax.

Neonatal pneumothorax usually occurs within the first 3 days of birth in late preterm and term newborns ${ }^{22,26)}$. Similarly, in the present study, all patients developed pneumothorax within the first 48 hours of life, with a mean onset time of 20.2 hours of life. These results were earlier than the onset time in early preterm infants born at $<32$ weeks of $\mathrm{GA}^{2,27)}$. It has been suggested that pneumothorax in late-preterm and full-term newborns is mainly related to mechanical injuries of the lungs caused by trans-pulmonary pressure to maintain sufficient functional residual capacity at the initiation of breathing. In the first few minutes of life, the trans-pulmonary pressures averaged $40 \mathrm{cmH}_{2} \mathrm{O}$, and occasional pressures as high as $100 \mathrm{cmH}_{2} \mathrm{O}$ were applied to the neonatal lung ${ }^{28)}$. The clinical presentation of neonatal pneumothorax is nonspecific. In the present study, more than three-quarters of the patients had increased oxygen demand to maintain appropriate saturation, and half of the patients had tachypnea before being diagnosed with pneumo thorax. In accordance with the results of the present study, a retrospective population-based descriptive study conducted in Orebro County reported that the common clinical presentations of neonatal pneumothorax were tachypnea (77\%) and cyanosis $(56 \%)^{24)}$. However, these respiratory symptoms and signs cannot be distinguished from those of other neonatal pulmonary diseases including RDS and TTN. Therefore, careful attention should be paid to the development of pneumothorax in late preterm and full-term infants within the first 3 days of life, and appropriate examinations, including chest radiography or lung ultrasound, should be performed immediately if respiratory symptoms worsen, such as aggravated tachypnea and increased oxygen demand.

Neonatal pneumothorax is associated with increased perinatal morbidities. In the present study, infants who developed pneumothorax had significantly higher rates of severe IVH. Few studies have reported that neonatal pneumothorax is significant ly associated with IVH and persistent pulmonary hypertension of the newborn (PPHN $)^{4,29)}$. These comorbidities were found to contribute to increased mortality in newborns with pneumothorax. Significantlyhigher mortality rates were observed in newborns who developed pneumothorax than in those without pneumothorax, in which mortality rates of neonatal pneumothorax have been reported to be between $16 \%$ and $65 \%^{22,29-31)}$. In the present study, the mortality rate of the pneumothorax group was $16.7 \%(6 / 36)$, which was statistically significant compared to that of the control group. However, the most common immediate cause of death was not pneumothorax but underlying pulmonary disease or concomitant neonatal morbidities ${ }^{22,29-31)}$. The deaths of the 
pneumothorax group in the present study were directly caused by unresponsive PPHN in three cases, severe IVH in two cases, and multi-organ dysfunction related to severe perinatal asphyxia in one case. Therefore, proper management of underlying pulmonary diseases and concomitant morbidities associated with pneumothorax is important to reduce mortality in infants with pneumothorax.

For early detection of neonatal pneumothorax, it is necessary to determine the risk factors associated with pneumothorax and identify high-risk groups. Zanardo et al. ${ }^{23)}$ found that elective cesarean section before 39 weeks of GA resulted in an increase in the incidence of neonatal pneumothorax. Many previous studies have focused on respiratory support, especially pressure support, during resuscitation or before the diagnosis of pneumothorax. A Canadian retrospective cohort study and a Japanese study showed that CPAP use during neonatal resuscitation was a risk factor for pneumothorax in late preterm and full-term neonates ${ }^{2,32)}$. A study on the birth cohort of the Parkland Health and Hospital System showed that the incidence rates of pneumothorax significantly increased in early full-term neonates after the introduction of CPAP in the neonatal resuscitation program ${ }^{33)}$. The high maximum peak inspiratory pressure during the 24 hours before the detection of pneumothorax and clinical procedures, including suction procedures and reintubation, have also been associated with the occurrence of pneumothorax ${ }^{29,31,34,35)}$. The present study found that the need for PPV in the delivery room and high $\mathrm{FiO}_{2}$ on admission were associated with neonatal pneumothorax with an OR of 3.40 (95\% CI, 1.26 to 9.12; $P=0.015$ ) and 1.06 (95\% CI, 1.03 to $1.09 ; P<0.001)$. Overall, these findings suggest that underlying lung disease with low compliance and interventions applying additional pressure above the average transpulmonary pressure during respiratory transition and damage to the airways and lungs caused by invasive procedures during the first few days of life were considered important riskfactors associated with pneumothorax in late preterm and full-term newborns with respiratory distress. Based on these findings, continuous monitoring of respiratory distress and follow-up chest radiography after birth are thought to help detect pneumothorax early in late preterm and term infants, who require advanced neonatal resuscitation (PPV, intubation, and chest compression) and relatively high oxygen concentration to achieve an $\mathrm{SPO}_{2}$ of $\geq 90 \%$. However, the findings of some studies on the risk factors affecting the development of pneumothorax in late preterm and full-term newborns are still inconsistent. Further large-scale and well-designed studies on the risk factors associated with neonatal pneumothorax are needed.

However, the present study has some limitations. First, this study had a retrospective design that depended on chart reviews, and the number of study populations was small because the present study was conducted in a single center. Second, chest radiography is not routinely performed in newborns without symptoms or signs of respiratory distress. The actual incidence of neonatal pneumothorax could have been underestimated due to the missing diagnosis of asymptomatic neonatal pneumothorax. Third, potential confounders included variations in neonatal resuscitation programs in the delivery room between inborn and out-born infants and insufficient data on the maternal or prenatal characteristics of out-born infants.

In conclusion, pneumothorax frequently develops in late preterm and full-term newborns with respiratory distress and is associated with high mortality and morbidity. Therefore, late preterm and full-term newborns with respiratory distress, especially those who required PPV to initiate breathing at birth and had relatively high oxygen concentrations to maintain appropriate oxygen saturation, should be meticulously followed up to monitor their respiratory conditions during their first 3 days of life. Furthermore, additional well-designed, prospective, controlled studies are needed to better predict and detect early neonatal pneumothorax in late preterm and full-term newborns with respiratory distress.

\section{ARTICLE INFORMATION}

\section{Ethical statement}

The institutional Review Board of the Kyungpook National University Chilgok Hospital approved the study (IRB No. 202110-003). Informed written consent was waived due to the retrospective design of the study.

\section{Conflicts of interest}

No potential conflict of interest relevant to this article was reported.

\section{Author contributions}

Conception and design: J.S.K.

Acquisition, analysis, or interpretation of data: E.A.K., J.H.J., S.Y.L., J.S.K. 
Drafting the work or revising: E.A.K., J.S.K.

Final approval of the manuscript: All authors read and approved the final manuscript.

\section{ORCID}

Eun-Ah Kim https://orcid.org/0000-0003-0788-8834

Jae-Hun Jung https://orcid.org/0000-0002-4980-0872

Sang-Yoon Lee https://orcid.org/0000-0003-2595-505X

Sook-Hyun Park https://orcid.org/0000-0002-4782-1127

Ji Sook Kim https://orcid.org/0000-0002-0100-5845

\section{Funding}

None

\section{Acknowledgments}

None

\section{REFERENCES}

1. Smith J, Schumacher RE, Donn SM, Sarkar S. Clinical course of symptomatic spontaneous pneumothorax in term and late preterm newborns: report from a large cohort. Am J Perinatol 2011;28:163-8.

2. Duong HH, Mirea L, Shah PS, Yang J, Lee SK, Sankaran K. Pneumothorax in neonates: trends, predictors and outcomes. J Neonatal Perinatal Med 2014;7:29-38.

3. Kitsommart R, Martins B, Bottino MN, Sant'Anna GM. Expectant management of pneumothorax in preterm infants receiving assisted ventilation: report of 4 cases and review of the literature. Respir Care 2012;57:789-93.

4. Trevisanuto D, Doglioni N, Ferrarese P, Vedovato S, Cosmi E, Zanardo V. Neonatal pneumothorax: comparison between neonatal transfers and inborn infants. J Perinat Med 2005;33: 449-54.

5. Hill A, Perlman JM, Volpe JJ. Relationship of pneumothorax to occurrence of intraventricular hemorrhage in the premature newborn. Pediatrics 1982;69:144-9.

6. Landry JS, Menzies D. Occurrence and severity of bronchopulmonary dysplasia and respiratory distress syndrome after a preterm birth. Paediatr Child Health 2011;16:399-403.

7. Ozer EA, Ergin AY, Sutcuoglu S, Ozturk C, Yurtseven A. Is pneumothorax size on chest $\mathrm{x}$-ray a predictor of neonatal mortality? Iran J Pediatr 2013;23:541-5.

8. El-Masry H, Aladawy MA, Sayed Younis MM, Mahmoud DH. Risk factors for pneumothorax in ventilated neonates admitt ed to neonatal intensive care unit (NICU). Ann Neonatol J
2021;3:70-87.

9. Terzic S, Heljic S, Panic J, Sadikovic M, Maksic H. Pneumothorax in premature infants with respiratory distress syndrome: focus on risk factors. J Pediatr Neonatal Individ Med 2016;5:e050124.

10. Girard I, Sommer C, Dahan S, Mitanchez D, Morville P. Risk factors for developing pneumothorax in full-term neonates with respiratory distress. Arch Pediatr 2012;19:368-73.

11. Zarogoulidis P, Kioumis I, Pitsiou G, Porpodis K, Lampaki S, Papaiwannou A, et al. Pneumothorax: from definition to diagnosis and treatment. J Thorac Dis 2014;6(Suppl 4):S372-6.

12. Lee AC, Panchal P, Folger L, Whelan H, Whelan R, Rosner B, et al. Diagnostic accuracy of neonatal assessment for gestational age determination: a systematic review. Pediatrics 2017;140: e20171423.

13. Schlaudecker EP, Munoz FM, Bardaji A, Boghossian NS, Khalil A, Mousa $\mathrm{H}$, et al. Small for gestational age: case definition \& guidelines for data collection, analysis, and presentation of maternal immunisation safety data. Vaccine 2017;35(48 Pt A): 6518-28.

14. Dubil EA, Magann EF. Amniotic fluid as a vital sign for fetal wellbeing. Australas J Ultrasound Med 2013;16:62-70.

15. Papile LA, Burstein J, Burstein R, Koffler H. Incidence and evolution of subependymal and intraventricular hemorrhage: a study of infants with birth weights less than 1,500 gm. J Pediatr 1978;92:529-34.

16. Thebaud B, Goss KN, Laughon M, Whitsett JA, Abman SH, Steinhorn RH, et al. Bronchopulmonary dysplasia. Nat Rev Dis Primers 2019;5:78.

17. Patel RM, Ferguson J, McElroy SJ, Khashu M, Caplan MS. Defining necrotizing enterocolitis: current difficulties and future opportunities. Pediatr Res 2020;88(Suppl 1):10-5.

18. Silva IS, Flor-de-Lima F, Rocha G, Alves I, Guimaraes H. Pneumothorax in neonates: a level III neonatal intensive care unit experience. J Pediatr Neonatal Individ Med 2016;5:e050220.

19. Kim Y, Lee E. Pneumothorax in full-term neonates transferred due to respiratory distress. Perinatology 2019;30:140-6.

20. Hadzic D, Skokic F, Husaric E, Alihodzic H, Softic D, Kovacevic D. Risk factors and outcome of neonatal pneumothorax in Tuzla Canton. Mater Sociomed 2019;31:66-70.

21. Walker MW, Shoemaker M, Riddle K, Crane MM, Clark R. Clinical process improvement: reduction of pneumothorax and mortality in high-risk preterm infants. J Perinatol 2002;22:641-5.

22. Vibede L, Vibede E, Bendtsen M, Pedersen L, Ebbesen F. Neonatal pneumothorax: a descriptive regional danish study. Neonatology 2017;111:303-8.

23. Zanardo V, Padovani E, Pittini C, Doglioni N, Ferrante A, Trevisanuto $\mathrm{D}$. The influence of timing of elective cesarean section on risk of neonatal pneumothorax. J Pediatr 2007;150:2525.

24. Andersson J, Magnuson A, Ohlin A. Neonatal pneumothorax: 
symptoms, signs and timing of onset in the post-surfactant era. J Matern Fetal Neonatal Med. 2021 Feb 3 [Epub]. https://doi.org/ 10.1080/14767058.2021.1882981.

25. Okumus M, Zubarioglu AU. Neonatal pneumothorax: 10 years of experience from a single center. J Pediatr Res 2020;7:163-7.

26. Apiliogullari B, Sunam GS, Ceran S, Koc H. Evaluation of neonatal pneumothorax. J Int Med Res 2011;39:2436-40.

27. Bhatia R, Davis PG, Doyle LW, Wong C, Morley CJ. Identification of pneumothoraxin very preterm infants. JPediatr 2011;159:11520.

28. Karlberg P, Cherry BR, Escardo FE, Koc G. Respiratory studies in newborn infants. II: Pulmonary ventilation and mechanics of breathing in the first minutes of life, including the onset of respiration. Acta Paediatr 1962;51:121-36.

29. Bhat Yellanthoor R, Ramdas V. Frequency and intensive care related risk factors of pneumothorax in ventilated neonates. Pulm Med 2014;2014:727323.

30. Navaei F, Aliabadi B, Moghtaderi M, Kelishadi R. Predisposing factors, incidence and mortality of pneumothorax in a neonatal intensive care unit in Isfahan, Iran. Zhongguo Dang Dai Er Ke Za Zhi 2010;12:417-20.

31. Watkinson M, Tiron I. Events before the diagnosis of a pneumothorax in ventilated neonates. Arch Dis Child Fetal Neonatal Ed 2001;85:F201-3.

32. Hishikawa K, Goishi K, Fujiwara T, Kaneshige M, Ito Y, Sago H. Pulmonary air leak associated with CPAP at term birth resuscitation. Arch Dis Child Fetal Neonatal Ed 2015;100:F3827.

33. Smithhart W, Wyckoff MH, Kapadia V, Jaleel M, Kakkilaya V, Brown LS, et al. Delivery room continuous positive airway pressure and pneumothorax. Pediatrics 2019;144:e20190756.

34. Niwas R, Nadroo AM, Sutija VG, Gudavalli M, Narula P. Malposition of endotracheal tube: association with pneumothorax in ventilated neonates. Arch Dis Child Fetal Neonatal Ed 2007; 92:F233-4.

35. Ngerncham S, Kittiratsatcha P, Pacharn P. Risk factors of pneumothorax during the first 24 hours of life. J Med Assoc Thai 2005;88 Suppl 8:S135-41. 